\title{
Validez factorial del "Cuestionario para la Evaluación del Síndrome de Quemarse por el Trabajo" (CESQT) en una muestra de médicos mexicanos"
}

\author{
Factorial Validity of the "Spanish Burnout Inventory" (SBI) \\ in a Sample of Mexican Doctors
}

Recibido: enero 8 de 2009

Revisado: marzo 18 de 2009

Aceptado: abril 26 de 2009

\author{
PEDRO R. GIL-MONTE** \\ Universidad de Valencia, España \\ Leslie Claret ZúNíga-CABAllero ${ }^{* * *}$ \\ Universidad Latina de América A.C., Morelia, México
}

* Artículo de investigación en psicometría.

** Dirección de contacto: Facultad de Psicología, Avda. Blasco Ibáñez, 21, 46010 Valencia, Tfno: 963864564 / Fax: 963864668, Correo electrónico: pedro.gil-monte@uv.es

**** Manantial Cointzio Norte \# 355 Fra. Los Manantiales, 58170 Morelia, Mich-México. Correo electrónico:1czuniga@unla.edu.mx

\section{RESUMEN}

El objetivo del estudio fue evaluar la validez factorial del Cuestionario para la Evaluación del Síndrome de Quemarse por el Trabajo (CESQT). Se hipotetizó un modelo de cuatro factores. La muestra estuvo compuesta por 110 médicos del Instituto Mexicano del Seguro Social. El instrumento está formado por 20 ítems distribuidos en 4 subescalas: Ilusión por el trabajo ( 5 ítems), Desgaste psíquico (4 ítems), Indolencia (6 ítems), y Culpa (5 ítems). El modelo presentó un ajuste adecuado: $\mathrm{GFI}=.83, \mathrm{RMSEA}=.061, \mathrm{NNFI}=.90$, $\mathrm{CFI}=.92, \mathrm{PNFI}=.65 . \mathrm{Los}$ resultados indican que el CESQT presentó en esta muestra validez factorial y valores de consistencia interna adecuados. Palabras clave autores

Salud ocupacional, síndrome de quemarse por el trabajo, análisis factorial, CESQT.

Palabras clave descriptor

Síndrome de burnout, análisis factorial, salud ocupacional.

\section{A B S T R A C T}

The purpose of this study was to assess the factorial validity of the "Spanish Burnout Inventory" (SBI). A model of four factors was hypothesized. The sample consisted of 110 doctors working in Mexican Institute of Social Security. The instrument is made up of 20 ítems, and 4 subscales: Enthusiasm toward the job (5 ítems), Psychological Exhaustion (4 ítems), Indolence (6 ítems), and Guilt (5 ítems). The measures of goodness of fit indicated that the hypothesized model showed a satisfactory fit for the data: GFI $=.83$, RMSEA $=.061, \mathrm{NNFI}=.90, \mathrm{CFI}=.92, \mathrm{PNFI}=.65$. As a whole, the results show that the SBI offers factorial validity and internal consistency to evaluate burnout for Mexican doctors.

Key words authors

Occupational Health, Factor Analysis, Burnout, SBI.

Key words plus

Burnout Psychology, Factor Analysis, Industrial Hygiene. 
El síndrome de quemarse por el trabajo (SQT) (burnout) es un riesgo laboral de carácter psicosocial que ha cobrado gran relevancia en las últimas décadas entre los profesionales del sector servicios que atienden a personas, y en especial en determinados colectivos, como los profesionales de la salud (Ortega \& López, 2004). Este fenómeno ha sido definido como una respuesta psicológica al estrés laboral crónico de carácter interpersonal y emocional que aparece en los profesionales de las organizaciones de servicios que trabajan en contacto con los clientes o usuarios de la organización. Se caracteriza por un deterioro cognitivo, consistente en la pérdida de la ilusión por el trabajo o la baja realización personal en él; por un deterioro afectivo caracterizado por agotamiento emocional y físico; y por la aparición de actitudes y conductas negativas hacia los clientes y hacia la organización en forma de comportamientos indiferentes, fríos, distantes, y lesivos. En ocasiones, estos síntomas se acompañan de sentimientos de culpa (Gil-Monte, 2005, 2008b).

El instrumento más utilizado para la evaluación del SQT ha sido el Maslach Burnout Inventory [MBI] (Maslach \& Jackson, 1986), que define el fenómeno como baja realización personal en el trabajo, alto agotamiento y alta despersonalización o cinismo (Maslach, Schaufeli \& Leiter, 2001). Aunque este instrumento ha obtenido valores adecuados de fiabilidad y validez en numerosos estudios, también se detectan con frecuencia insuficiencias psicométricas. Por ejemplo, valores relativamente bajos para la subescala de Despersonalización, una estructura factorial del modelo con fundamento teórico diferente de la ofrecida por el manual, o la ambigüedad factorial de algunos ítems que con frecuencia presentan cargas factoriales superiores a .40 en más de un factor (Gil-Monte, 2005; Halbesleben \& Demerouti, 2005; Kristensen, Borritz, Villadsen \& Christensen, 2005). Las insuficiencias se acentúan cuando se adapta a idiomas diferentes al inglés (Olmedo, Santed, Jiménez \& Gómez, 2001; Peeters \& Rutte, 2005; Truchot, Keirsebilck \& Meyer, 2000).

Estas insuficiencias han generado la necesidad de construir instrumentos de evaluación alternativos (Halbesleben \& Demerouti, 2005; Kristensen et al., 2005). Es el caso del Cuestionario para la Evaluación del Sindrome de Quemarse por el Trabajo [CESQT] (Gil-Monte, 2005; Gil-Monte et al., 2006). El CESQT está formado por cuatro dimensiones denominadas: 1. Ilusión por el trabajo, definida como el deseo del individuo de alcanzar las metas laborales porque supone una fuente de realización personal; 2. Desgaste psíquico, definida como la aparición de agotamiento emocional y físico debido a que en el trabajo se tiene que tratar a diario con personas que presentan o causan problemas; 3. Indolencia, definida como la aparición de actitudes negativas de indiferencia y cinismo hacia los clientes de la organización; 4. Culpa, definida como la aparición de sentimientos de culpa por el comportamiento y las actitudes negativas desarrolladas en el trabajo, en especial hacia las personas con la que se establecen relaciones laborales (Gil-Monte, 2005).

Los ítems se construyeron tras una revisión de la literatura, y a partir de los resultados de entrevistas realizadas a individuos que habían desarrollado el SQT en algún grado. Siguiendo el protocolo de entrevista de Sackman (1992), se obtuvieron un conjunto de síntomas, causas y desencadenantes del SQT, así como una descripción cualitativa del proceso. Los síntomas fueron sometidos a un análisis en el que se etiquetaron como emociones, $\operatorname{cog}$ niciones, o actitudes. A partir de esta clasificación se elaboraron un total de 74 ítems. En su elaboración se tuvo presente la delimitación semántica del ítem y los aspectos de redacción y comprensión adecuada (Meliá, 2000). Posteriormente, fueron sometidos a análisis estadísticos para determinar su validez discriminante, su distribución factorial, y su contribución a la consistencia interna de la escala en la que se integraron. Tras una serie de sucesivos estudios con muestras de diferentes colectivos profesionales (v.g., sanidad, atención a personas con discapacidad, docentes) (Caro, Gil-Monte \& Gonçalves, 2005; Gil-Monte, 2008a; Gil-Monte et al., 2006), se retuvieron aquellos elementos que presentaron repetidamente valores de asimetría dentro del rango $+/$ - 1 , una distribución factorial adecuada con cargas factoriales superiores a .40 
en el factor en el que debían agruparse y cargas factoriales inferiores a .30 en el resto de factores, y que incrementaban la consistencia interna alfa de Cronbach de la dimensión en la que fueron incluidos.

El modelo teórico que subyace al CESQT considera que el SQT es una respuesta al estrés laboral crónico, característica de los profesionales que trabajan con personas. En este modelo, el deterioro cognitivo (bajas puntuaciones en ilusión por el trabajo) y afectivo (altas puntuaciones en desgaste psíquico) aparece en un primer momento como respuesta a las fuentes de estrés laboral crónico y, con posterioridad, los individuos desarrollarán actitudes negativas hacia las personas que atienden en su trabajo (altos niveles de indolencia). La aparición de los sentimientos de culpa es posterior a estos síntomas, pero no la presentan todos los individuos. De esta manera, es posible distinguir dos perfiles en el proceso del SQT. El perfil 1 conduce a la aparición de un conjunto de sentimientos y conductas vinculados al estrés laboral que originan una forma moderada de malestar, pero que no incapacita al individuo para el ejercicio de su trabajo, aunque podría realizarlo mejor. Este perfil se caracteriza por la presencia de baja ilusión por el trabajo, junto a altos niveles de desgaste psíquico e indolencia, pero los individuos no presentan sentimientos de culpa. El perfil 2 constituye con frecuencia un problema más serio que identificaría a los casos clínicos más deteriorados por el desarrollo del SQT. Además de los síntomas anteriores los individuos presentan también sentimientos de culpa (Gil-Monte, 2005, 2008b).

En estudios previos, mediante análisis factorial exploratorio (AFE) se han obtenido resultados adecuados de validez factorial para el CESQT y de consistencia interna para sus escalas con muestras de profesionales de enfermería en España (GilMonte, 2008a). El cuestionario ha sido adaptado a otras culturas, obteniendo también resultados adecuados de validez factorial mediante AFE y validez concurrente con el MBI en Chile (Olivares \& Gil-Monte, 2007). También han sido adecuados los resultados sobre la consistencia interna de las subescalas en estudios realizados en Argentina (Marucco, Gil-Monte \& Flamenco, 2007), Chile (Olivares \& Gil-Monte, 2007), España (GilMonte, 2008a; Gil-Monte et al., 2006), México (Unda, Sandoval \& Gil-Monte, 2007), y Portugal (Figueiredo-Ferraz, Gil-Monte \& Grau-Alberola, 2009).

El análisis factorial confirmatorio (AFC) puede ser utilizado en la validación de cuestionarios para evaluar la validez de constructo en los casos en que un investigador tiene suficientes conocimientos previos, para formular hipótesis sobre la relación entre indicadores y dimensiones latentes (BatistaFoguet, Coenders \& Alonso, 2004). El AFC aporta evidencia empírica sobre la estructura interna de una prueba psicométrica. Mediante AFC se ha confirmado la estructura factorial del CESQT y su validez de constructo en estudios realizados en España con profesionales que trabajan hacia personas con discapacidad (Gil-Monte, 2008a; Gil-Monte et al., 2006), y en México (Gil-Monte, Unda \& Sandoval, 2009) y Portugal (Gil-Monte $\&$ Figueiredo-Ferraz, sometido) con muestras de maestros.

El CESQT ofrece algunas ventajas frente a otros instrumentos existentes, entre las más relevantes cabe señalar que: a) parte de un modelo teórico previo al modelo psicométrico; b) si bien algunas dimensiones son similares a las del Maslach Burnout Inventory [MBI-HSS] (Maslach \& Jackson, 1986), incorpora los sentimientos de culpa como un síntoma que permite establecer diferentes perfiles en la evolución del SQT, e incluye ítems que evalúan aspectos cognitivos y físicos del agotamiento, además de los emocionales, y c) supera las insuficiencias psicométricas señaladas arriba derivadas de la adaptación al castellano de otros instrumentos utilizados para evaluar el fenómeno.

El objetivo de este estudio es analizar la validez factorial del CESQT en una muestra de médicos mexicanos. Se hipotetiza que el modelo de cuatro factores que reproduce el modelo original alcanzará un ajuste adecuado. 


\section{Método}

\section{Participantes}

La muestra del estudio estuvo compuesta por 110 médicos del Instituto Mexicano del Seguro Social con un amplio rango de especialidades (médico familiar y no familiar, pediatra, internista, cirujano, oncólogo, ginecólogo, traumatólogo, dermatólogo, etc.).

Según el sexo, 63 individuos se identificaron como hombres $(57.80 \%)$ y 46 como mujeres $(42.20$ $\%)$. La media de edad de la muestra fue de 46.22 años $(\mathrm{dt}=7.90$, max. $=64$, min. $=25)$. Según el tipo de contrato, el $84.50 \%$ de los participantes tenía contrato indefinido, mientras que el 13.60 $\%$, contrato temporal. El $1.80 \%$ no respondió esta pregunta. La media de antigüedad en la profesión fue de 19.47 años $(d t=7.60)$, la media de antigüedad en la organización estuvo en 16.91 años ( $\mathrm{dt}=$ 7.44), y la media de antigüedad en el puesto fue de 13.87 años $(\mathrm{dt}=8.33)$.

\section{Instrumentos}

Los datos fueron recogidos mediante el Cuestionario para la Evaluación del Síndrome de Quemarse por el Trabajo, versión para profesionales de la salud (CESQT-PS). El cuestionario consta de 20 ítems que se distribuyen en cuatro subescalas denominadas: Ilusión por el trabajo (5 ítems) (v.g.: Veo mi trabajo como una fuente de realización personal), Desgaste psíquico (4 ítems) (v.g.: Me siento desgastada/o emocionalmente), Indolencia (6 ítems) (v.g.: No me apetece atender a algunos pacientes), y Culpa (5 ítems) (v.g.: Tengo remordimientos por algunos de mis comportamientos en el trabajo). Los ítems se evalúan mediante una escala de frecuencia de 5 grados, que va de 0 (Nunca) a 4 (Muy frecuentemente: todos los días), correspondiendo al punto intermedio una puntuación de 2 ("A veces: algunas veces al mes"). Bajas puntuaciones en Ilusión por el trabajo junto a altas puntuaciones en Desgaste psíquico e Indolencia suponen altos niveles del SQT.

\section{Procedimiento}

Los datos fueron recogidos mediante selección no aleatoria y voluntaria de los participantes, en las que se garantizó la confidencialidad de las respuestas. El análisis de los datos se realizó mediante el paquete estadístico SPSS 15. Para realizar el análisis factorial confirmatorio (AFC) los datos fueron analizados con el programa LISREL 8.30 (Jöreskog \& Sörbom, 1996). El método de estimación fue Máxima Verosimilitud (ML).

Para valorar el ajuste global del modelo, además del índice $\mathrm{Chi}^{2}$ se consideraron otros índices que no están afectados por el tamaño de la muestra, y permiten evaluar diferentes aspectos del modelo hipotetizado y su ajuste a los datos. Así, el Goodness of Fit Index (GFI) mide la cantidad relativa de varianza explicada por el modelo. El Non-Normed Fit Index (NNFI) es un indicador del ajuste relativo del modelo. El Comparative Fit Index (CFI) indica el grado de ajuste del modelo cuando se compara con un modelo nulo. Para estos índices valores superiores a .90 se consideran indicadores de un ajuste aceptable del modelo (Bentler, 1992; Hoyle, 1995). El Root Mean Square Error of Aproximation (RMSEA) estima la cantidad global de error existente en el modelo. Valores entre .05 y .08 indican un ajuste adecuado del modelo (Browne \& Cudeck, 1993; Hair, Anderson, Tatham \& Black, 1995). El Parsimony Normed Fit Index (PNFI) evalúa el grado de complejidad del modelo. Valores superiores a .50 se consideran adecuados cuando los índices de ajuste (GFI, NNFI) presentan valores superiores a .90 (Byrne, 1998).

\section{Resultados}

Los resultados estadísticos sobre el análisis de ítems y subescalas se presentan en la Tabla 1. Se observa que las medias más elevadas se obtuvieron para los ítems que forman la dimensión Ilusión por el trabajo (ítems, 1, 5, 10, 15, y 19) que se caracterizan porque altas puntuaciones indican bajos niveles del SQT. Por el contrario, las medias más bajas corresponden a los ítems de Indolencia y Culpa. 
Con respecto al grado de asimetría se observa que los ítems de la escala de Ilusión por el trabajo presentaron una asimetría negativa moderada, siendo para el resto de elementos más baja, excepto el ítem 11, que evalúan Indolencia, que presentó una asimetría positiva más elevada que el resto de elementos de la subescala. Para todos los ítems la homogeneidad corregida alcanzó valores superiores a .30, con excepción del ítem 11 que alcanzó un valor de .23. Los ítems, con excepción del ítem 11 , contribuyeron a incrementar el coeficiente alfa de Cronbach de la subescala de la que formaban parte (Tabla 1). Estos valores, unidos al contenido semántico del ítem, permiten afirmar que todos los ítems están relacionados significativamente con aquellos construidos para evaluar la misma faceta del síndrome, formando parte del mismo constructo.

TABLA 1

Análisis estadístico de los ítems

\begin{tabular}{|c|c|c|c|c|}
\hline Subescala / Ítem & $\mathrm{M}(\mathrm{dt})$ & Homogeneidad corregida & Asimetría & Alfa sin ítem \\
\hline Ilusión por el trabajo $(\alpha=0.72)$ & $3.58(.56)$ & & -1.76 & \\
\hline 1 & $3.55(.83)$ & 0.34 & -2.20 & 0.67 \\
\hline 5 & $3.74(.66)$ & 0.55 & -2.99 & 0.60 \\
\hline 10 & $3.51(1.02)$ & 0.41 & -2.16 & 0.65 \\
\hline 15 & $3.75(.55)$ & 0.63 & -2.43 & 0.59 \\
\hline 19 & $3.36(1.06)$ & 0.43 & -1.83 & 0.65 \\
\hline Desgaste psíquico $(\alpha=0.86)$ & $1.48(.92)$ & & .49 & \\
\hline 8 & $2.09(1.22)$ & 0.61 & -0.12 & 0.86 \\
\hline 12 & $1.35(1.15)$ & 0.73 & 0.46 & 0.80 \\
\hline 17 & $1.39(1.02)$ & 0.77 & 0.41 & 0.79 \\
\hline 18 & $1.09(1.01)$ & 0.71 & 0.85 & 0.81 \\
\hline Indolencia $(\alpha=0.75)$ & $.71(.55)$ & & .90 & \\
\hline 2 & $1.03(.92)$ & 0.58 & .94 & 0.67 \\
\hline 3 & $.87(.85)$ & 0.56 & 1.08 & 0.68 \\
\hline 6 & $.58(.70)$ & 0.59 & 1.12 & 0.68 \\
\hline 7 & $.59(.64)$ & 0.54 & .83 & 0.69 \\
\hline 11 & $.38(.83)$ & 0.23 & 2.74 & 0.77 \\
\hline 14 & $.83(.99)$ & 0.44 & 1.44 & 0.72 \\
\hline Culpa $(\alpha=0.79)$ & $.72(.61)$ & & 1.10 & \\
\hline 4 & $1.02(1.23)$ & 0.44 & 1.33 & 0.78 \\
\hline 9 & $.82(.98)$ & 0.67 & 1.69 & 0.65 \\
\hline 13 & $.51(.65)$ & 0.63 & 1.32 & 0.69 \\
\hline 16 & $.67(.65)$ & 0.42 & 0.85 & 0.74 \\
\hline 20 & $.60(.67)$ & 0.62 & 0.86 & 0.69 \\
\hline
\end{tabular}

Fuente: elaboración propia. 
Respecto a las medias de las subescalas, el valor más alto se obtuvo para Ilusión por el trabajo $(\mathrm{M}=$ $3.58, \mathrm{dt}=.56)$. Y el valor más bajo se obtuvo para la subescala de Indolencia $(\mathrm{M}=.71 \mathrm{dt}=.55)$, con un valor similar al valor de la media obtenido para la subescala de Culpa $(\mathrm{M}=.72, \mathrm{dt}=.61)$.

El modelo obtuvo un ajuste adecuado para la muestra con varios índices de ajuste del modelo considerados, excepto con el GFI que obtuvo un valor inferior a .90 (Byrne, 1998) (Tabla 2). Con estos valores se puede concluir que el modelo de ecuaciones estructurales presentó un ajuste global suficientemente adecuado y se confirma la hipótesis formulada. Debido a que la prueba $\mathrm{Chi}^{2}$ es sensible al tamaño de la muestra, se calculó la razón entre su valor y los grados de libertad. Existe consenso en la literatura de que valores inferiores a 2 indican un ajuste adecuado del modelo (Kline, 1998), por tanto el valor obtenido en este estudio indica que el modelo ajusta $\left(\mathrm{Chi}^{2} / \mathrm{gl}=1.37\right)$. Aunque la cantidad relativa de varianza explicada por el modelo (GFI $=.83$ ) fue insuficiente, el ajuste del modelo resultó adecuado al considerar el error de aproximación a los valores de la matriz de covarianza de la población (RMSEA $=.061)$, y también según los índices de ajuste relativo del modelo (NNFI $=.90$, y CFI $=$.92) (Byrne, 1998). El PNFI también presentó un valor adecuado (PNFI $=.65)$ (Byrne, 1998).

Todas las cargas factoriales resultaron significativas (Figura 1). El parámetro más bajo se obtuvo para el elemento 11 del cuestionario, que pertenece a la subescala de Indolencia. El parámetro para este elemento alcanzó un valor de $\lambda=.25$ $(t=2.47)$.
En la Figura 1 se observa que todas las correlaciones entre las dimensiones del CESQT resultaron significativas para $p<.05$. Como era de esperar, según la definición de las dimensiones, las relaciones entre Ilusión por el trabajo y el resto de dimensiones del CESQT resultaron negativas, mientras que las relaciones entre las restantes dimensiones resultaron positivas. La relación más intensa se estableció entre Indolencia y Culpa (.76, $\mathrm{p}<.05)$, y la menos intensa entre Ilusión por el trabajo y Desgaste psíquico $(-.29, \mathrm{p}<.05)$, lo que permite afirmar que estas dos dimensiones no son estimaciones inversas del mismo constructo.

El coeficiente alfa de Cronbach alcanzó valores superiores a .70 para todas las subescalas del CESQT. Los valores obtenidos fueron: Ilusión por el trabajo, alfa $=.72$; para Desgaste psíquico, alfa $=.86$; para Indolencia, alfa $=.75 ;$ y para Culpa, alfa $=.79$.

\section{Discusión}

El objetivo de este artículo ha sido analizar la validez factorial del CESQT en una muestra de médicos mexicanos para concluir sobre la validez transnacional de este instrumento en profesionales de la salud. Con base en los resultados del estudio, se puede concluir que el CESQT, en su versión para profesionales de la salud (CESQT-PS), resulta un instrumento suficientemente fiable y válido para evaluar el SQT en médicos mexicanos. Los resultados de los índices de ajuste global del modelo a los datos, han confirmado la estructura factorial hipotetizada. Este resultado es similar a

TABLA 2

Índices de ajuste global para el modelo factorial hipotetizado

\begin{tabular}{|c|c|c|c|c|c|c|c|}
\hline $\mathrm{Chi}^{2}$ & $\mathrm{p}$ & gl & $\mathrm{Chi}^{2} / \mathrm{gl}$ & GFI & RMSEA & NNFI & CFI \\
\hline 225.06 & .001 & 164 & 1.37 & 0.83 & 0.061 & 0.90 & 0.92 \\
\hline
\end{tabular}

Nota: GFI = Goodness Fit Index, RMSEA = Root Mean Square Error of Approximation, NNFI = NonNormed Fit Index, CFI = Comparative Fit Index, PNFI = Parsimony Normed Fit Index.

Fuente: elaboración propia. 


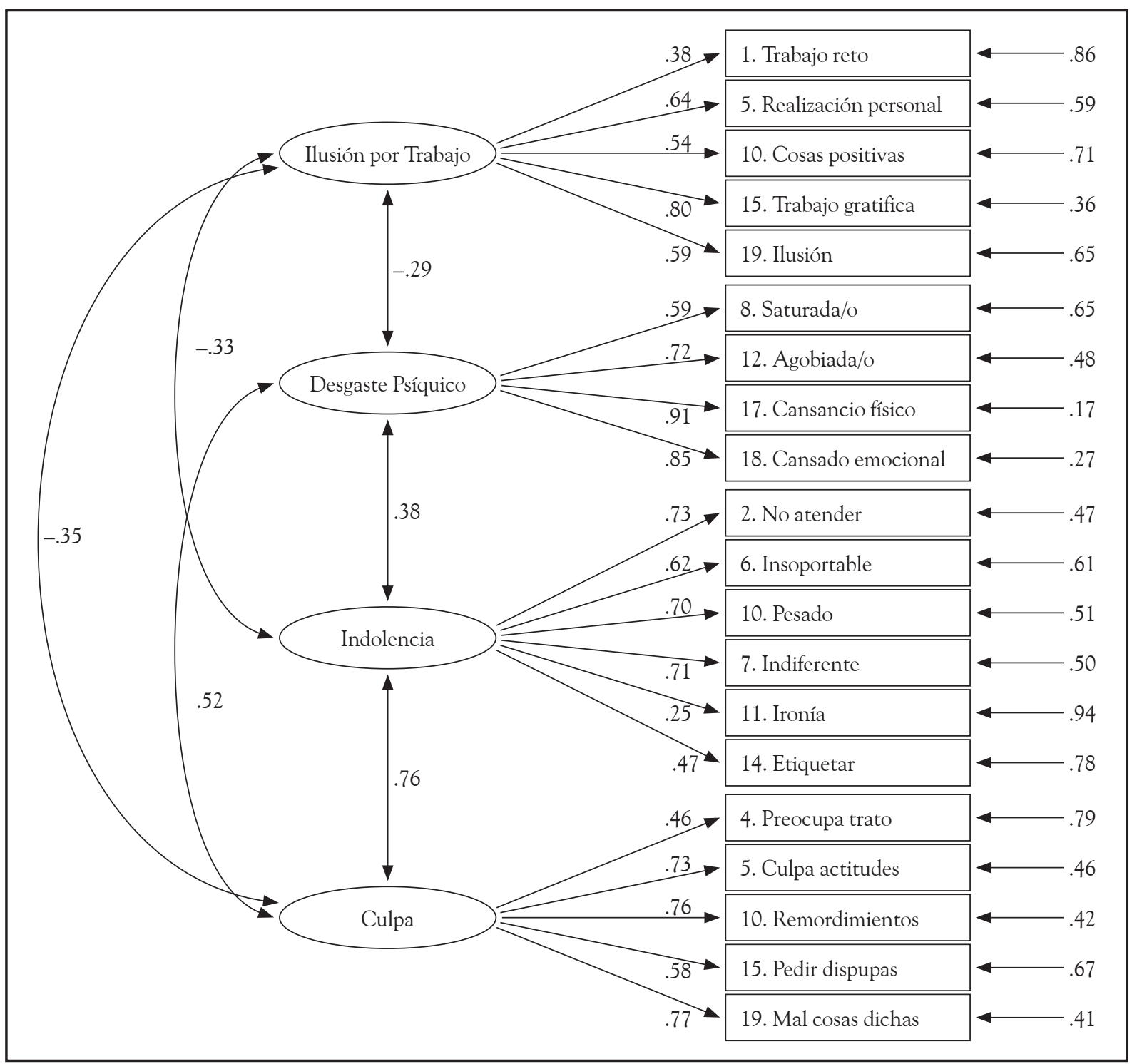

FIGURA 1

Resultados del modelo factorial hipotetizado para el CESQT-PS.

Fuente: elaboración propia.

los obtenidos en estudios previos mediante análisis factorial exploratorio y confirmatorio (Gil-Monte, 2008a; Gil-Monte et al., 2006; Olivares \& GilMonte, 2007), lo que contribuye a la validación del instrumento y a la validación psicométrica del modelo teórico de cuatro dimensiones, origen del cuestionario.

Las saturaciones factoriales resultaron adecuadas, con los ítems contribuyendo a la consistencia interna de la subescala de la que forman parte. Una excepción aparece en el ítem 11 (Me apetece ser irónico/a con algunos pacientes), de la escala de Indolencia, que presentó un carga factorial y un valor de homogeneidad corregida inferior a .30 para ambos parámetros, al tiempo que su eliminación incrementaba el alfa de Cronbach de la escala. No obstante, según la opinión de expertos emitida durante el proceso de adaptación del cuestionario 
a poblaciones mexicanas, se concluyó que el contenido semántico del ítem era comprensible y aplicable, por lo que se mantuvo la formulación original del elemento y su inclusión en la escala. Por otra parte, este elemento ha obtenido valores adecuados en los parámetros referidos en estudios previos realizados en México con maestros (GilMonte et al., 2009).

Los resultados para este ítem también han sido adecuados en otros colectivos ocupacionales de la salud, en estudios realizados en España (GilMonte, 2008a) y en Portugal (Caro et al., 2005) con profesionales de enfermería. Estos resultados nos llevan a afirmar que la falta de ajuste del ítem a la escala, puede ser una insuficiencia del instrumento en este estudio, pero no generalizable a su adaptación a la cultura mexicana, o a colectivos profesionales de la salud.

Todas las escalas han alcanzado valores de fiabilidad alfa de Cronbach adecuados (CarreteroDios \& Pérez, 2007). Por tanto, se puede concluir que el CESQT-PS reúne los suficientes requisitos de consistencia interna como para ser empleado en la estimación del SQT en médicos mexicanos.

Como recomendaciones para seguir trabajando en la validación del modelo teórico del CESQT y en su estructura factorial se recomienda: a) realizar estudios que repliquen los resultados obtenidos con trabajadores de diferentes colectivos ocupacionales, en diferentes países, y en diferentes contextos socioculturales; b) también es importante realizar estudios que contribuyan a la identificación de criterios de clasificación de los individuos en las dimensiones del cuestionario para concluir sobre la prevalencia e incidencia del SQT, y de esta manera poder diseñar planes de intervención; c) una tercera vía de trabajo viene determinada por la necesidad de conocer mejor cómo progresa el SQT (Taris, Le Blanc, Schaufeli \& Schreurs, 2005). El modelo teórico que subyace al CESQT (Gil-Monte, 2005) puede aportar información en esta línea, por ello se recomienda realizar estudios longitudinales que analicen empíricamente la relación antecedentes-consecuentes entre las dimensiones del cuestionario, y entre éstas y sus antecedentes y consecuencias.
Entre las implicaciones para la práctica derivadas de la utilización del CESQT, cabe señalar que es un instrumento de evaluación psicométrica que puede facilitar el diagnóstico de individuos que han desarrollado el SQT en diferentes culturas. El instrumento está diseñado desde un modelo teórico que incluye los sentimientos de culpa, y que diferencia dos formas en la evolución del SQT, con repercusiones diferentes para la salud mental de los individuos (Gil-Monte, 2005). Este diagnóstico diferencial puede ser importante para los profesionales, pues ayuda a entender comportamientos diferentes de individuos con altos niveles del síndrome.

\section{Referencias}

Batista-Foguet, J. M., Coenders, G. \& Alonso, J. (2004). Análisis factorial confirmatorio. Su utilización en la validación de cuestionarios relacionados con la salud. Medicina Clínica, 122, 21-27.

Bentler, P. M. (1992). On the fit of models to covariances and methodology to the Bulletin. Psychological Bulletin, 112, 400-404.

Browne, M. W. \& Cudeck, R. (1993). Alternative ways of assessing model fit. En K. A. Bollen \& L. S. Long (Eds.), Testing Structural Equation Models (pp. 136 162). Newbury Park (CA): Sage.

Byrne, B. M. (1998). Structural equation modeling with LISREL, PRELIS, and SIMPLIS: Basic concepts, applications, and programming. London: Lawrence Erlbaum Associates.

Caro, M., Gil-Monte, P. R. \& Gonçalves, S. (2005, junio). O burnout em profissionais de enfermagen: un estudo transcultural para a validaçâo do questionario "CESQT". Estudio presentado en el I Congresso Nacional Saúde e Comportamento, Braga, Portugal.

Carretero-Dios, H. \& Pérez, C. (2007). Standards for the development and review of instrumental studies: Considerations about test selection in psychological research. International Journal of Clinical and Health Psychology, 7, 863-882.

Figueiredo-Ferraz, H., Gil-Monte, P. R. \& Grau-Alberola, E. (2009). Prevalencia del Síndrome de que- 
marse por el trabajo (burnout) en una muestra de maestros portugueses. Aletheia, 29, 6-15.

Gil-Monte, P. R. (2005). El sindrome de quemarse por el trabajo (burnout): una enfermedad laboral en la sociedad del bienestar. Madrid: Pirámide.

Gil-Monte, P. R. (2008a). Evaluación psicométrica del síndrome de quemarse por el trabajo (burnout): el cuestionario "CESQT". En J. Garrido (Comp.), iMaldito trabajo! (pp. 269-291). Barcelona: Granica.

Gil-Monte, P. R. (2008b). Magnitude of relationship between burnout and absenteeism: A preliminary study. Psychological Reports, 102, 465-468.

Gil-Monte, P. R. \& Figueiredo-Ferraz, H. (sometido). Psychometric properties of the "Spanish Burnout Inventory" (SBI) among Portuguese teachers.

Gil-Monte, P. R., García-Juesas, J. A., Núñez, E., Carretero, N., Roldán, M. D. \& Caro, M. (2006). Validez factorial del "Cuestionario para la Evaluación del Síndrome de Quemarse por el Trabajo". Psiquiatria.com, 10. Recuperado el 8 de enero, 2009, de www.psiquiatria.com/articulos/ psicosis/24872/? + + interactivo

Gil-Monte, P. R., Unda, S. \& Sandoval, J. I. (2009). Validez factorial del "Cuestionario para la Evaluación del Síndrome de Quemarse por el Trabajo" (CESQT) en una muestra de maestros mexicanos. Salud Mental, 31, 205-214.

Halbesleben, J. R. \& Demerouti, E. (2005). The construct validity of an alternative measure of burnout: Ivestigating the English translation of the Oldenburg Burnout Inventory. Work \& Stress, 19, 208-220.

Hair, J. H., Anderson, R. E., Tatham, R. L. \& Black, W. C. (1995). Multivariate Data Analysis (4⿳亠丷⿵冂丶 ed.). Englewood Cliffs, NJ: Prentice-Hall.

Hoyle, R. H. (1995). The Structural Equation Modeling approach: Basic concepts and fundamental issues. En R. H. Hoyle (Ed.), Structural Equation Modeling: Concepts, Issues, and Applications (pp. 1-15). Thousand Oaks, CA: Sage.

Jöreskog, K. G. \& Sörbom, D. (1996). LISREL 8: User's reference guide. Chicago: Scientific Software International.

Kline, R. B. (1998). Principles and practice of structural equation modelling. New York: Guildford Press.
Kristensen,T. S., Borrritz, M., Villadsen, E. \& Christensen, K. B. (2005). The Copenhagen Burnout Inventory: A new tool for the assessment of burnout. Work \& Stress, 19, 192-207.

Marucco, M. A, Gil-Monte, P. R. \& Flamenco, E. (2007). Síndrome de quemarse por el trabajo (burnout) en pediatras de hospitales generales, estudio comparativo de la prevalencia medida con el MBI-HSS y el CESQT. Informació Psicològica, 91/92, 32-42.

Maslach, C. \& Jackson, S. E. (1986). Maslach Burnout Inventory (2 ${ }^{-}$ed). Palo Alto, CA: Consulting Psychologists Press.

Maslach, C., Schaufeli, W. B. \& Leiter, M. P. (2001). Job burnout. Annual Review of Psychology, 52, 397-422.

Meliá, J. L. (2000). Teoría de la fiabilidad y de la validez. Valencia: Cristóbal Serrano.

Olivares, V. E. \& Gil-Monte, P. R. (2007). Análisis de las propiedades psicométricas del "Cuestionario para la Evaluación del Síndrome de Quemarse por el Trabajo" (CESQT) en profesionales chilenos. Ansiedad y Estrés, 13, 229-240.

Olmedo, M., Santed, M. A, Jiménez, R. \& Gómez, M. D. (2001). El síndrome de burnout: variables laborales, personales y psicopatológicas asociadas. Psiquis. 22, 117-29.

Ortega, C. \& López, F. (2004). El burnout o síndrome de estar quemado en los profesionales sanitarios: revisión y perspectivas. International Journal of Clinical and Health Psychology, 4, 137-160.

Peeters, M. A. \& Rutte, C. G. (2005). Time management behavior as a moderator for the Job Demand-Control interaction. Journal of Occupational Health Psychology, 10, 64-75.

Sackmann, S. A. (1992). Culture and subcultures: An analysis of organizational knowledge. Administrative Science Quarterly, 37, 140-161.

Taris, T. W., Le Blanc, P. M., Schaufeli, W. B. \& Schreurs, P. J. (2005). Are there causal relationships between the dimensions of the Maslach Burnout Inventory? A review and two longitudinal tests. Work \& Stress, 19, 238-55.

Truchot, D., Keirsebilck, L. \& Meyer, S. (2000). Communal orientation may not buffer burnout. Psychological Reports, 86, 872-78. 
Unda, S., Sandoval, J. I. \& Gil-Monte, P. R. (2007). Prevalencia del síndrome de quemarse por el trabajo (SQT) (burnout) en maestros mexicanos. Informació Psicológica, 91/92, 53-63. 